\title{
The E166 Experiment: Undulator-Based Production of Polarized Positrons
}

\author{
A. Mikhailichenko ${ }^{b}$, G. Alexander ${ }^{j}$, Y. Batygin ${ }^{i}, S$. Berridge ${ }^{\mathrm{k}}$, \\ V. Bharadwaj ${ }^{i}$, G. Bower ${ }^{j}$, W. Bugg ${ }^{k}$, F.-J. Decker ${ }^{i}$, R. Dollan ${ }^{\mathrm{g}}$, \\ Y. Efrimenkok, V. Gharibyan ${ }^{\mathrm{e}}, \mathrm{C}$. Hast ${ }^{\mathrm{i}}$, R. Iverson', \\ H. Kolanoski ${ }^{\mathrm{g}}$, J. Kovermann ${ }^{\mathrm{a}}$, K. Laihem ${ }^{\mathrm{f}}$, T. Lohse ${ }^{\mathrm{g}}$, \\ K.T. McDonald ${ }^{\text {h, G.A. Moortgat-Pick }}{ }^{\text {, }}$ P. Pahl ${ }^{\mathrm{e}}$, R. Pitthan, \\ R. Pöschl ${ }^{e}$, E. Reinherz-Aronis ${ }^{j}$, S. Riemann ${ }^{f}, A . S_{\text {Schälicke }}{ }^{f}$, \\ K.P. Schüler ${ }^{e} *$, T. Schweizer ${ }^{\mathrm{g}}$, D. Scott ${ }^{\mathrm{c}}$, J.C. Sheppard ${ }^{\mathrm{i}}$, \\ A. Stahl ${ }^{a}, Z$. Szalata, D. Walz ${ }^{i}$, A. Weidemann ${ }^{i}$ \\ ${ }^{a}$ RWTH Aachen, D-52056 Aachen, Germany \\ ${ }^{b}$ Cornell University, Ithaca, NY 14853, USA \\ ${ }^{\circ}$ CCLRC Daresbury Laboratory, Daresbury, Warrington, Cheshire, WA4 4AD, UK \\ ${ }^{d}$ University of Durham, Durham, DH1 3HP, UK \\ ${ }^{e}$ DESY, D-22607 Hamburg, Germany \\ ${ }^{f}$ DESY Zeuthen, D-15738 Zeuthen, Germany \\ ${ }^{8}$ Humboldt University, D-12489 Berlin, Germany \\ ${ }^{h}$ Princeton University, Princeton, NJ 08544, USA \\ ${ }^{i} S L A C$, Menlo Park, CA 94025, USA \\ ${ }^{j}$ University of Tel Aviv, Tel Aviv 69978, Israel \\ ${ }^{k}$ University of Tennessee, Knoxville, TN 37996, USA
}

\begin{abstract}
A proof-of-principle experiment has been carried out in the Final Focus Test Beam (FFTB) at SLAC to demonstrate production of polarized positrons in a manner suitable for implementation at the ILC. A helical undulator of $2.54 \mathrm{~mm}$ period and $1-\mathrm{m}$ length produced circularly polarized photons of first harmonic endpoint energy of $8 \mathrm{MeV}$ when traversed by a $46.6 \mathrm{GeV}$ electron beam. The polarized photons were converted to polarized positrons in a 0.2-radiation-length tungsten target. The polarization of these positrons was measured at several energies, with a peak value of $\approx 80 \%$ according to a preliminary analysis of the transmission polarimetry of photons obtained on reconversion of the positrons in a second tungsten target.
\end{abstract}

Keywords: ILC, polarized positron source, helical undulator, Compton transmission polarimeter PACS: $29.27 . \mathrm{Hj}, 41.60 .-\mathrm{m}, 41.75 . \mathrm{Fr}$,

* corresponding author: Peter.Schuler@desy.de

CP915, Proceedings of the $17^{\text {ih }}$ International Spin Physics Symposium, edited by K. Imai, T. Murakami, N. Saito, and K. Tanida 2007 American Institute of Physics 978-0-7354-0423-6/07/\$23.00

1095 


\section{Introduction}

An important requirement for the ILC will be the development of an intense positron beam that matches the bunch charge and current of the electron beam. Conventional technology, as used at the SLC, in which positrons are generated directly by electrons of several GeV in showers on a thick tungsten target, would be quite challenged to cope with target damage and activation issues associated with the considerably higher average beam currents that are required at the ILC. Furthermore, a conventional positron source cannot generate polarized positrons.

In the scheme proposed by Balakin and Mikhailichenko [1] a helical undulator is employed to generate photons of several MeV with circular polarization which are then converted in a relatively thin target to produce longitudinally polarized positrons. Positrons generated in thin targets suffer less from multiple scattering and can therefore be collected with greater efficiency for subsequent acceleration. As the undulator is not expected to diminish the emittance of the drive beam in a significant way, one can directly use the primary high-energy electron beam of the $\mathrm{ILC}$, either at full energy or at an intermediate energy of $150 \mathrm{GeV}$, to generate the undulator photons for positron production. However, in order to obtain the required high production rate of more than one positron per parent electron, a truly long undulator with a length of at least $100 \mathrm{~m}$ would be required at the ILC [2].

The purpose of the E166 experiment [3] was to explore the potential of this concept with a short scaled-down undulator structure in the final focus test beam (FFTB) at SLAC. Of primary importance is the degree of positron polarization that can be achieved. Particular emphasis was therefore placed on the polarimetry of the positrons. In addition we measured also the polarization of the undulator photon beam.

The important role of beam polarization at e+e-colliders was demonstrated with polarized electrons at the SLC. Even more powerful physics investigations can be carried out if both electrons and positrons are polarized [4]. If a positron beam polarization of about $60 \%$ can be realized, it will be possible to enhance or suppress to a substantial degree individual processes for different helicity configurations.

\section{The E166 Experiment}

\section{General Description}

A schematic layout of the experiment is given in Fig. 1. The high-energy electron beam passes through the undulator and is then deflected with a chain of dump magnets (DM) into the beam dump (not shown). The photon beam generated in the undulator is analyzed by photon transmission polarimetry [3]. Positrons are generated by pair production in a 0.2 r.l. tungsten production target (T1) and deflected with a doubledipole spectrometer into the positron analyzer. A solenoid lens (SL) behind the production target helps to increase the useful positron flux. A second tungsten target (0.5 r.1.) in front of the positron analyzer reconverts positrons to photons (T2). The positron polarization is inferred from the polarization of the reconverted photons which is also measured by transmission polarimetry. AG1 and AG2 are identical 
aerogel flux counters with $\sim 5 \mathrm{MeV}$ threshold, AG1Si and AG2Si : associated silicon detectors. GCAL : silicon/tungsten photon calorimeter, PosSi : segmented positron flux detector, CsI : Cesium-Iodide calorimeter detector behind the positron analyzer, C1-C4 : photon collimators, J : movable jaws in the positron spectrometer.

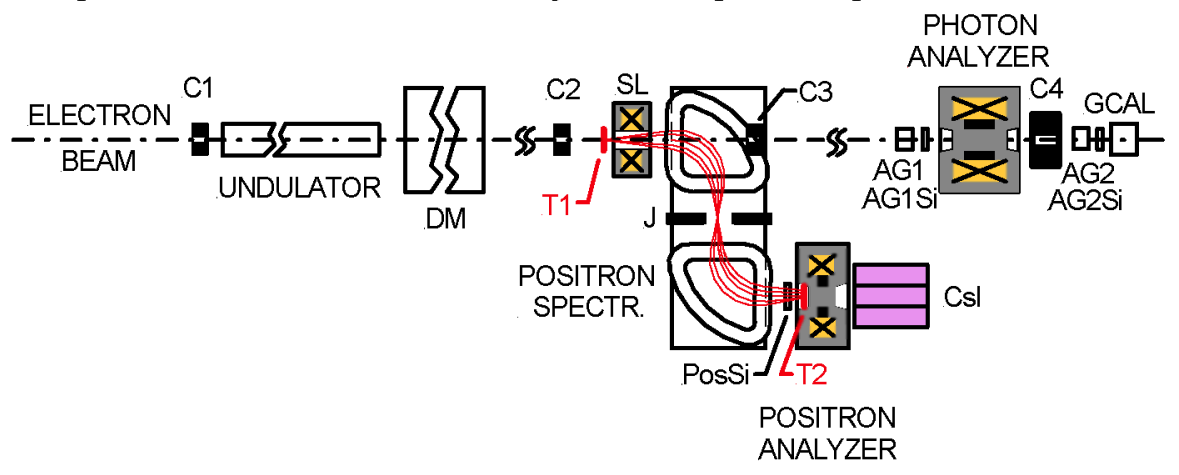

FIGURE 1. Schematic layout of the E166 experiment (not to scale).

\section{Helical Undulator}

The helical undulator employed in E166 has a length of 1 meter with a period of $2.54 \mathrm{~mm}$ and a clear aperture of only $0.9 \mathrm{~mm}$. With an electron beam energy of 46.6 $\mathrm{GeV}$, it generates circularly polarized photons of energy up to $8 \mathrm{MeV}$, with energy spectrum and polarization appropriate for the ILC, only the intensity of the photons is lower in the demonstration experiment.
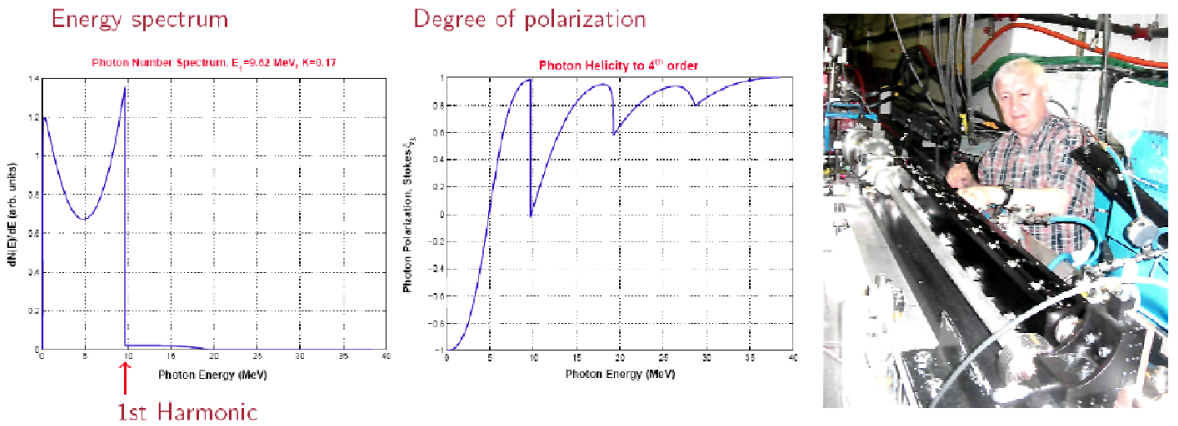

FIGURE 2. (a) Undulator photon spectrum $\mathrm{dN} / \mathrm{dE}$, integrated over angle, calculated for an electron beam energy $\mathrm{E}_{\mathrm{e}}=50 \mathrm{GeV}$, undulator period $\lambda_{\mathrm{u}}=2.4 \mathrm{~mm}$, and undulator strength parameter $\mathrm{K}=0.17$ which gives a peak energy $\mathrm{E}_{1}=9.62 \mathrm{MeV}$ for the first harmonic (dipole) radiation. Actual parameters were slightly different: $\mathrm{E}_{\mathrm{e}}=46.6 \mathrm{GeV}, \lambda_{\mathrm{u}}=2.54 \mathrm{~mm}, \mathrm{~K}=0.19, \mathrm{E}_{1}=8.0 \mathrm{MeV}$.

(b) Photon polarization, calculated for the same conditions as for the shown spectrum.

(c) The helical undulator during installation in the FFTB tunnel at SLAC. 


\section{Analyzer Magnets}

Two analyzer magnets were employed for the measurement of the photon and positron beam polarization. Both are iron core solenoids with core dimensions of 50 $\mathrm{mm} \varnothing \times 150 \mathrm{~mm}$ long for the photon analyzer, and $50 \mathrm{~mm} \varnothing \times 75 \mathrm{~mm}$ long for the positron analyzer. The shape of the structure (Fig. 3) was modeled to obtain saturation magnetization over most of the cylindrical core volume except for the end regions.
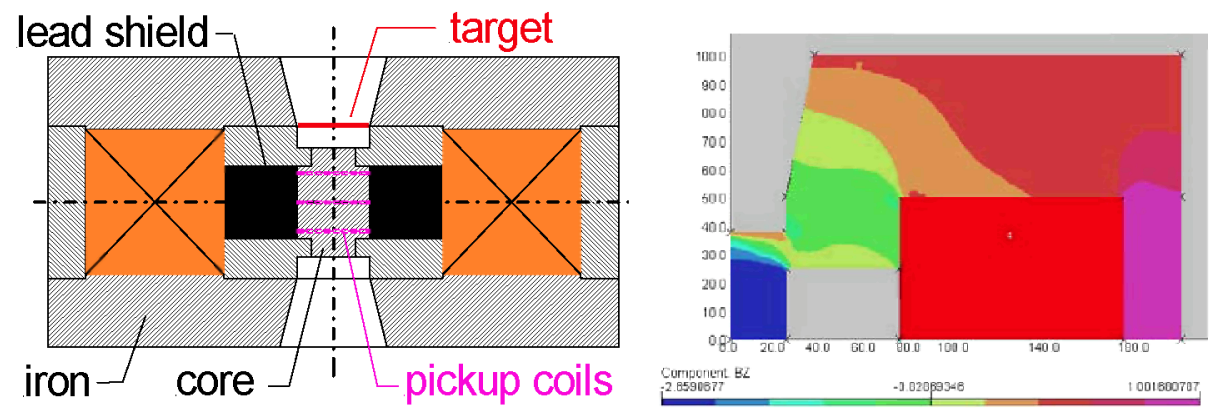

FIGURE 3. Analyzer magnet for positron polarimetry: (a) full cross section (left figure) (b) $2 \mathrm{~d}$-field modeling (right figure) shown for one of four identical quadrants.

The electron polarization of the iron is given by $P_{e}=M_{s} /\left(\rho_{e} \mu_{B}\right)$, where $M_{s}=$ $\left(M_{s} / M\right) M$ is the dominant spin part of the total magnetization $M, \rho_{e}$ the electron density and $\mu_{\mathrm{B}}$ the Bohr magneton. $\mathrm{M}_{\mathrm{s}} / \mathrm{M}=0.958 \pm 0.002$ is known from EinsteindeHaas type experiments. We determined the saturation magnetization $M$ from flux measurements with pickup coils in the central core region (Fig. 4) and used the field modeling to account for the drop-off at the ends. All in all, we obtain a preliminary value of $\mathrm{P}_{\mathrm{e}}=0.0694 \pm 0.0017$ for the electron polarization of the iron.

Positron Analyzer Magnet (Center)

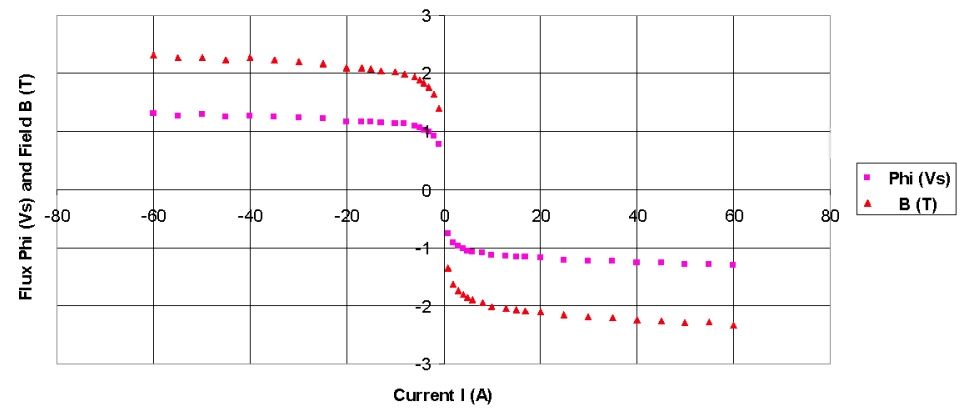

FIGURE 4. Flux and field vs. current in the positron analyzer magnet, as measured in one of several pickup coils surrounding the iron core. Polarization asymmetries were measured at \pm 60 amperes with the iron in full saturation over most of its core length. 


\section{Results}

The experiment collected data during June and September 2005. All of the results reported here are based on $10 \mathrm{~Hz}$ beam pulse frequency. The positron signal in the PosSi silicon detector was optimized for each of 6 different spectrometer settings by scanning the current of the focusing solenoid lens. Figure 5 shows the beam pulse integrated photon signals in the CsI detector array from converted positrons. Electron beam halo showering in the undulator structure caused a relatively high background level (undulator off) in the CsI detector. Background was continuously recorded by delaying the undulator pulse timing for every other beam pulse.

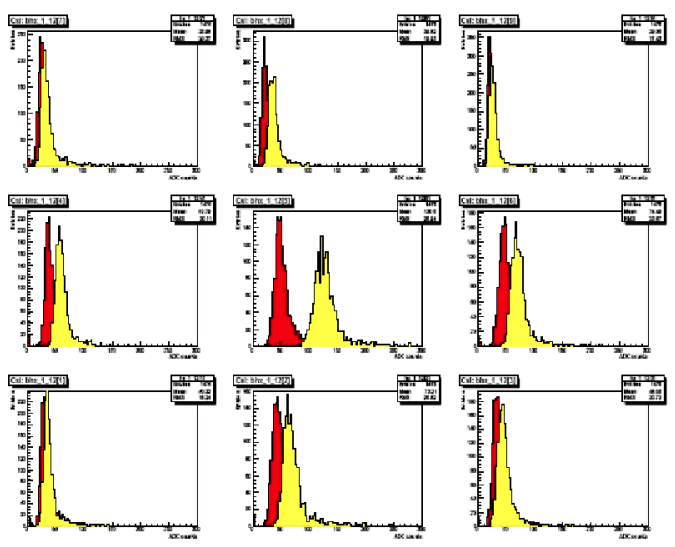

FIGURE 5. Calorimeter signal + background (light color) and background (dark color) distributions in the $3 \times 3$ CsI crystal array. Background was measured for every other beam pulse by delaying the undulator pulse by several microseconds. One $\mathrm{ADC}$ count is approximately $1.7 \mathrm{MeV}$.

The magnetization direction of the analyzer magnets was reversed every 3000 machine pulses $(5$ minutes at $10 \mathrm{~Hz}$ ). Figure 6 shows background subtracted signals and asymmetries in the central CsI crystal for a typical run segment. Data were taken for six different energy settings of the spectrometer.

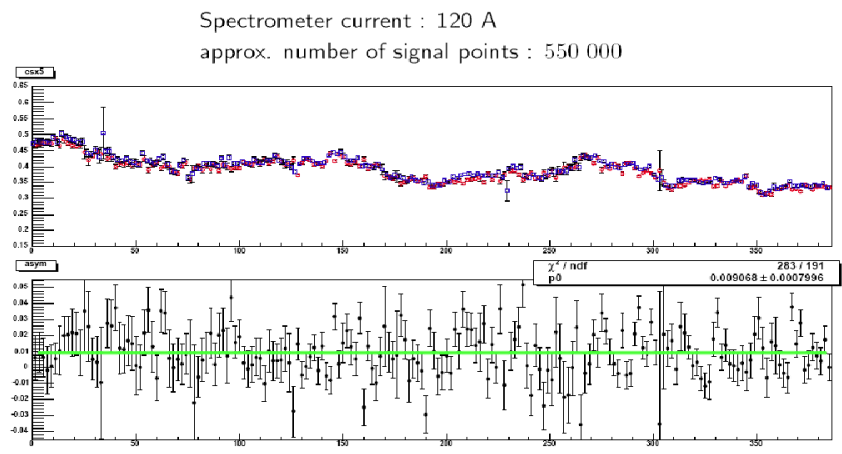

FIGURE 6. Background subtracted central CsI signals and asymmetries for a typical run segment. 
TABLE 1. Preliminary E166 Results: Measured Positron Asymmetries and Polarization Values

\begin{tabular}{|c|c|c|c|c|c|c|}
\hline $\begin{array}{c}\text { spectrometer } \\
\text { current } \\
(\mathrm{A})\end{array}$ & $\begin{array}{c}\text { positron } \\
\text { energy } \\
(\mathrm{MeV})\end{array}$ & $\begin{array}{c}\text { measured } \\
\text { asymmetry } \\
\delta(\%)\end{array}$ & $\begin{array}{c}\text { statistical } \\
\text { error } \\
\Delta \delta(\%)\end{array}$ & $\begin{array}{c}\text { analyzing } \\
\text { power } \\
\mathbf{A}_{\mathbf{e}+}(\%)\end{array}$ & $\begin{array}{c}\mathbf{P}_{\mathbf{e}} \\
(\%)\end{array}$ & $\begin{array}{c}\text { positron } \\
\text { polarization } \\
\mathbf{P}_{\mathbf{e}^{+}}(\%)\end{array}$ \\
\hline 100 & 3.7 & 0.57 & 0.17 & 19.0 & 7.0 & $43 \pm 13$ \\
\hline 120 & 4.5 & 0.88 & 0.10 & 18.0 & 7.0 & $70 \pm 9$ \\
\hline 140 & 5.2 & 1.03 & 0.08 & 16.8 & 7.0 & $88 \pm 9$ \\
\hline 150 & 5.6 & 0.78 & 0.10 & 16.0 & 7.0 & $70 \pm 10$ \\
\hline 160 & 6.0 & 0.83 & 0.12 & 15.8 & 7.0 & $75 \pm 12$ \\
\hline 180 & 6.7 & 0.89 & 0.21 & 15.8 & 7.0 & $80 \pm 20$ \\
\hline 180, ferrofl. & 6.7 & 0.98 & 0.21 & 15.8 & 7.0 & $89 \pm 20$ \\
\hline
\end{tabular}

Preliminary positron results for the central CsI crystal are given in Table 1. The asymmetry $\delta=\left(\mathrm{S}_{+}-\mathrm{S}_{-}\right) /\left(\mathrm{S}_{+}+\mathrm{S}_{-}\right)$of background subtracted calorimeter signals (normalized to beam current) is listed together with the analyzing power $\mathrm{A}_{\mathrm{e}+}$ (from simulations) and the electron polarization of the iron $\mathrm{P}_{\mathrm{e}}$. The polarization $\mathrm{P}_{\mathrm{e}}$ of the positrons (Fig. 7) is then obtained from the relation $\delta=\mathrm{A}_{\mathrm{e}}+\mathrm{P}_{\mathrm{e}} \mathrm{P}_{\mathrm{e}+}$. Only statistical errors are shown, but these are dominating and total errors should therefore be close. The results are consistent with a peak positron polarization of $80 \%$. The ongoing data analysis will be extended to all nine calorimeter crystals and will also include an electron measurement that was obtained with a reversed spectrometer field. We have also measured the undulator photon asymmetry and found it in good agreement with the expected value.

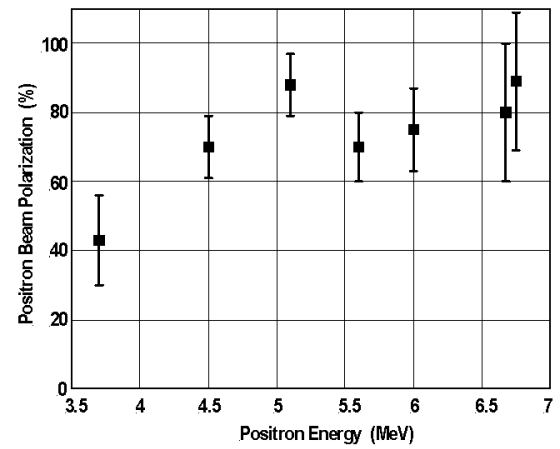

FIGURE 7. Preliminary E166 Results: Positron Beam Polarization

\section{REFERENCES}

1. V.E. Balakin and A.A. Mikhailichenko, "The Conversion System for Obtaining Highly Polarized Electrons and Positrons", Budger Institute of Nuclear Physics, Preprint BINP 79-85 (1979).

2. K. Flöttmann, "Investigation toward the Development of Polarized and Unpolarized High Intensity Positron Sources for Linear Colliders", DESY-93-161, Thesis, Universität Hamburg, 1993.

3. SLAC Proposal E166, "Undulator-Based Production of Polarized Positrons", June 2003.

4. G. Moortgat-Pick et al., "The role of polarized positrons and electrons in revealing fundamental interactions at the Linear Collider", hep-ph/0507011, July 2006. 